\title{
The Role of Functional and Dysfunctional Negative Emotions, Resilience and Mindfulness in Well-Being
}

\author{
B Shahzad and J Collard* \\ School of Psychology, The Cairnmillar Institute, Australia
}

Submission: August 01, 2019; Published: September 09, 2019

*Corresponding author: James Collard, School of Psychology, The Cairnmillar Institute, Melbourne, Australia

\section{Abstract}

This study sets out to investigate the binary model of emotions. It explores the relationships between suggested functional negative emotions and dysfunctional negative emotions with mindfulness, resilience, and well-being. The study was based on a sample of 104 adult participants. Results indicated that participants differentiated between proposed functional and dysfunctional negative emotion categories. Results of the structural equation modelling (SEM) demonstrated that functional negative emotions and dysfunctional negative emotions uniquely contributed to reduced well-being. The relationship of functional negative emotions to well-being was mediated by participants' resilience levels. The relationship of dysfunctional negative emotions to well-being was mediated by participants' resilience and mindfulness levels. SEM results indicated partial mediation, as the direct effect of functional and dysfunctional negative emotions on well-being was still substantial, though considerably reduced, after the intervention of resilience and mindfulness. The overall findings of the study supported the binary model of distress.

Keywords: Binary Model of Emotion; Mindfulness; Resilience; Well-Being

\section{Introduction}

Research has shown that during and after stressful experiences individuals' interpretations of events and their emotional and behavioural responses, seem to play an important role in their subjective well-being [1]. For instance, two people may experience similar problematic situations, yet one person may cope effectively and seem to be minimally affected, or even positively influenced, while the other person may respond less effectively and experience more psychological distress as a result [1]. There are several factors that are suggested to play a role in determining the degree to which people are successfully able to handle challenging situations. Included amongst these factors are the types of emotions that they experience [2], the level of skill they have at noticing, accepting and responding mindfully to their emotions [3], and their ability to show resilience and bounce back after a difficult situation [4]. Building on this, research has shown that strategies that diminish extreme efforts to control and regulate negative emotions and that encourage the acceptance of uncomfortable inner experiences provide a more adaptive approach to emotion regulation $[3,5]$.

The current study sets out to investigate Ellis's binary theory of emotions by exploring how emotions, suggested to fall under the func tional negative emotion and dysfunctional negative emotion categories, are related to the constructs of mindfulness, resilience and mental well-being.

\section{The Binary model of emotions}

The dominant model of emotion at present provides a unitary, continuum-based view of emotions. It proposes that dysfunctional negative emotions (i.e. anxiety and depression) are different from functional negative emotions (i.e., concern and sadness) simply on a quantitative basis [6]. In the unitary model dysfunctional emotions are more intense and distressing than functional emotions. It further proposes that the suggested increase in intensity acts to inhibit an individual's adaptation to their environment [7].

In contrast, Ellis [2] proposed that emotions that are triggered in response to irrational beliefs regarding negative events are dysfunctional negative emotions and emotions that follow rational beliefs regarding negative events are functional negative emotions. Following from this, he suggested that variations exist in intensity between many related emotions (e.g. depressed vs. sad, angry vs. annoyed) and that 


\section{Psychology and Behavioral Science International Journal}

emotions can also be separated based on their qualitative properties. About qualitative differences, the binary model of emotions advocates that the functionality of people's emotions can be determined according to their subjective experiences, related perceptions (e.g. if their beliefs are rational or irrational), and the behavioural consequences of the emotion [8].

In the binary model, functional negative emotions are suggested to involve a subjective experience that may be considered unpleasant, but it will generally be tolerable, and the behavioural consequences are suggested to be adaptive. For dysfunctional negative emotions, the subjective experience of the emotion is suggested to be negative and intolerable, and that the behavioural consequences are maladaptive as well i.e., the applied behaviour strategies are typically a form of dysfunctional coping about the emotion and/or the situation [8]. Table 1 provides a summary of the suggested functional and dysfunctional negative emotions.

Table 1: Inappropriate and appropriate negative emotions and their cognitive correlates.

\begin{tabular}{|c|c|c|c|}
\hline Inferences Related to Personal Domain & Type of Belief & Emotion & Appropriateness of Emotion \\
\hline \multirow{2}{*}{ Threat or danger } & Irrational & Anxiety & Inappropriate \\
\hline & Rational & Concern & Appropriate \\
\hline \multirow{2}{*}{ Loss (with implications for future): failure } & Irrational & Depression & Inappropriate \\
\hline & Rational & Sadness & Appropriate \\
\hline \multirow{2}{*}{$\begin{array}{c}\text { Breaking of personal rule (other or self); other } \\
\text { threatens self; frustration }\end{array}$} & Irrational & Damning anger & Inappropriate \\
\hline & Rational & Non damning anger (or annoyance) & Appropriate \\
\hline \multirow{2}{*}{ Breaking of own moral code } & Irrational & Guilt & Inappropriate \\
\hline & Rational & Remorse & Appropriate \\
\hline \multirow{2}{*}{ Other betrays self (self no-deserving) } & Irrational & Hurt & Inappropriate \\
\hline & Rational & Disappointment & Appropriate \\
\hline \multirow{2}{*}{ Threat to desires exclusive relationship } & Irrational & Morbid Jealousy & Inappropriate \\
\hline & Rational & Nonmorbid Jealousy & Appropriate \\
\hline \multirow{2}{*}{ Personal weakness revealed publicly } & Irrational & Shame & Inappropriate \\
\hline & Rational & Regret & Appropriate \\
\hline
\end{tabular}

This difference between functional and dysfunctional emotions can be illustrated with an example of a person providing a presentation at their work. If they approach the task with irrational cognitions (e.g., I must succeed, or I am a failure) the theory proposes that they will then experience dysfunctional emotions (e.g., anxiety while trying to achieve a goal and depression after failing to reach a goal). Such dysfunctional emotions would then contribute to not only an avoidable negative subjective experience but also to potentially unsuitable behavioural consequences (e.g. avoidance, safety behaviours) and excessively heightened physiological responses. If instead they approach the task with rational and flexible cognitions (e.g., I would love to succeed, if not it is not the end of the world), these are suggested to result in adaptive emotions (e.g. concern while trying to achieve a certain goal and sadness after failure). Such emotions are thought to result in tolerable subjective experiences, with physiological components that may even help to promote functional responses, and to promote behaviours that help with goal attainment.

Supporting the binary model of emotion, Kassinove, Eckhardt \& Endes [9] found that people did not identified differences in the intensity of emotions, based on a theoretical separation of functional and dysfunctional emotions. This challenges the unitary model of emotion, where the different emotional labels would be expected to be related to differing intensity of experience. In terms of functional differences between the emotions, the initial findings have tended to provide support for the binary model of emotions. Research has shown that the suggested dysfunctional negative emotions are related to clinically significant difficulties (e.g. depressive disorders, anxiety disorders) whereas, the suggested functional negative emotions (e.g. concern and sadness) relate to normal negative reactions by people confronting challenging situations [8]. Building on this David et al. [10] demonstrated that lower levels of irrational beliefs were related to functional emotions while high irrational beliefs (e.g. "I should be comfortable at all times") and catastrophizing were related with dysfunctional negative emotions and reduced energy levels. Similarly, Mogoase \& Stefan [11] demonstrated that dysfunctional emotions, as a combination of both functional and dysfunctional emotions, were related to irrational beliefs [11].

These research findings support Ellis' theory that there is a qualitative difference between functional and dysfunctional emotions. In line with these findings, the binary model of distress understands and recognizes the relevance of functional negative emotions and suggests focusing interventions primarily on reducing dysfunctional emotions [10].

\section{Mindfulness and well-being}

Mindfulness can be described as a psychological state of consciously becoming aware of and focusing on the existing moment [12-14]. It also holds the concept of acceptance towards one's internal experiences as central [15]. As such, it is described as the skill of being self-aware 
while maintaining a mental state where one feels free of any certain way of thinking or judging [16]. As a result, this is thought to promote the ability to accept and experience emotions without attempts to modify, avoid or control them [3,17]. This increased tolerance and acceptance of emotional states is then thought to increase efficacy, allowing thoughtful responses that promote adaptive movement towards one's goals $[18,19]$.

Mindfulness is often promoted as an alternative to the maladaptive emotional control strategy of suppression. Suppression aims to decrease the intensity and frequency of unwanted emotions [3], but paradoxically tends to lead to an escalation in the frequency of negative emotions and distress [20,21]. Unlike suppression, mindfulness aims to achieve regulated behaviour by shifting the focus from altering internal emotional states to changing one's connection to these states [20].

The suggested benefits of mindfulness have been supported in previous research that showed that individuals with higher levels of train mindfulness manage strong emotions without becoming either emotionally overwhelmed or emotionally distant [22]. This has, in part, has been attributed to the awareness component of mindfulness. It has also demonstrated associations with improved emotion regulation [23] and effective coping during stressful situations [24]. Research has also indicated that a reduction in awareness of the present moment and a judgemental attitude toward one's internal experiences may restrict an individual's adaptive learning and can intensify emotional responses [25].

Further evidence supporting the role of mindfulness in managing emotional responses comes from applied research, where mindfulness interventions are thought to enhance trait mindfulness. For example, Frye \& Spates [26] demonstrated that mindfulness strategies helped patients with post-traumatic stress disorder to confront trauma related stimuli for prolonged exposure, assisting in their recovery. Similarly, Eifert \& Forsyth [27] found that individuals with a range of anxiety disorders exhibited a reduction in avoidance behaviours and anxiety symptoms, along with improved quality of life, following training in acceptance and mindfulness skills. Others have also found that mindfulness meditation improves effective emotion regulation, reduced reactive responses [28,29] and reduced rigidity in cognitions [30,31].

Such findings are in line with the growing body of evidence that has shown mental health improvements from mindfulness-based stress reduction (MBSR) training, as compared to controls. Those receiving such training reported greater reductions in stress, anxiety, rumination, depressive symptoms, negative affect and physical illness and greater improvements in quality of life, emotional intelligence, social connectedness, attention and immune reactivity [32-36]. Together, this literature indicates that higher levels of mindfulness are associated with improved subjective well-being through cognitive flexibility, improved emotional functioning, and more functional behavioural choices.

\section{Resilience and well-being}

Resilience is the ability to get back up after a failure or a disappointment. It includes the ability to effectively regulate emotions and to adapt to the changing requirements of a stressful situation $[4,37,38]$. Five essential characteristics of resilience include meaningful life or purpose, perseverance, equanimity, self-reliance and existential aloneness [39]. There is debate as to whether people have inherent levels of resilience [40] or whether resilience can be nurtured and developed [41].

Research into resilience has shown that as people age and develop they tend to learn to deal more effectively with negative emotions in tense situations [41]. It has also been shown that individuals who are more resilient tend to extract positive coping strategies from difficult situations [40]. For example, they may consider a setback to be a learning experience or take a positive meaning out of a difficult situation. In support of this, Tugade \& Fredrickson [24] found that highly resilient people experienced more positive emotions, assessed anxiety provoking tasks as less threatening, recovered faster from negative emotional arousal, and acted more optimistically in negative situations than those with low levels of resilience. On the other hand, the individuals who were less resilient were found to struggle more in regulating negative emotions and tended to exhibit intensified reactivity towards stressful tasks [24].

It has also been suggested that resilient individuals intentionally produce and foster their positive emotions by practicing relaxation methods and focusing on positive thoughts [42]. These findings do not mean, however, that highly resilient people disregard the complexity of a situation. Research has indicated that such people still experience tension, get frustrated, and identify negative aspects of stressful situations [24]. These points were demonstrated in a study of widows. It was found that the widows who were highly resilient experienced a variety of positive (happy, calm, and joyful) and negative (restless, anxious, and miserable) emotions throughout the bereavement process [43]. Comparatively however, they presented with a higher level of life satisfaction and with less psychological distress than those who were less resilient.

One factor suggested to contribute to the development of resilience is mindfulness. It has been suggested that as a follow on from its ability to expand emotional awareness is the development of greater resilience and adaptation to stressful situations [44]. This was supported in a study by Orzech et al. [45] who found that mindfulness training was associated not only with reductions in stress and anxiety, but also with better subjective well-being and resilience. Elsewhere, it has also been suggested that mindfulness strategies can strengthen resilience and to prevent relapse with depression [46]. In summary, resilience has been shown to influence how people respond and adapt to challenging situations. While it does not stop people from experiencing negative emotions, it appears to influence the overall balance of their emotional reaction, with those lower on resilience experiencing greater distress.

\section{Present Research}

Research investigating Ellis' binary model of emotion is limited to date. Clinically, it appears relevant to gain a better understanding of the qualitative and intensity differences between functional and dysfunc- 
tional negative emotions. It is also important to understand how they relate to resilience and mindfulness, and how these constructs predict well-being. In line with the binary model, the literature suggests that dysfunctional negative emotions would have a stronger, negative relationship with well-being than that demonstrated by functional negative emotions. This is likely to be through differing relationships with mindfulness and resilience. Both mindfulness and resilience have demonstrated that while they do not prevent the experience of negative emotions, those reporting higher levels of each tend to report lower levels of distress and engage in more functional behaviours. Based on the literature reviewed, it is possible that this may be a result of mindfulness imparting protection against the expression of dysfunctional emotions. Thus, the examination of these relationships could better inform the understanding of distress and to further develop treatment approaches for distress (e.g., in tense situations, professionals may focus on dysfunctional negative emotions such as anxiety and depression, rather than focusing on functional negative emotions like concern and sadness).

\section{Aim and Hypotheses}

The aim of the present study is to investigate how functional and dysfunctional negative emotions, mindfulness, resilience and subjective well-being relate to one another. Based on the previous research it is hypothesized that:

i. There will be significant qualitative difference between functional negative emotions and dysfunctional negative emotions as reflected in the factorial structure of the respective scales' items.

ii. Dysfunctional negative emotions will significantly predict well-being with higher levels of dysfunctional negative emotions predicting low levels of well-being; and mindfulness and resilience will in series mediate the relationship between dysfunctional negative emotions and well-being.

iii. Functional negative emotions will significantly predict well-being with higher levels of functional negative emotions predicting lower levels of well-being; and resilience will mediate the relationship between functional negative emotions and well-being.

\section{Method}

\section{Participants}

Participants were recruited via internet postings using Facebook and email lists. There were 104 participants, with 65 females (62.5\%) and 39 males (37.5\%). They were aged between 19 and $65(\mathrm{M}=32.0$, $\mathrm{SD}=8.1)$.

\section{Measures}

Functional and dysfunctional negative emotions scale
(FADNES)

This is a 10-item questionnaire used to evaluate the experience of functional and dysfunctional negative emotional states experienced in the previous week. Each item has an option of four responses i.e. one functional emotion (e.g. concerned), one dysfunctional emotion (such as, anxious) an option of both (e.g. I felt both concerned and anxious) and a indicating that neither of the emotions was experienced (i.e., "I have not been feeling this way"). Participants are then asked to indicate the intensity of the experienced emotion on a 4-point Likert scale ranging from ( 1 = 'A little' to 4 = 'very much'). Examples of the emotional pairings include sadness/depression, concern/anxiety, annoyance/ anger, and regret/guilty the internal consistency of FADNES has been shown to range from good to excellent with alpha values ranging from .58 (functional subscale) to .81 (functional \& dysfunctional subscale; [11].

\section{Five facet mindfulness questionnaire-short form (FFMQ-sf)}

The FFMQ-sf is a 24-item questionnaire which assesses five different aspects of mindfulness i.e. observe, describe, act with awareness, non-judgment of internal experience, and non-reactivity to internal experience. Participants had to rate to the point to which several statements were true for them on a 5-point Likert scale from 1 (never true) to 5 (very often true). Higher scores on the scale indicate greater mindfulness. The scale has demonstrated good internal reliability with alpha values ranging from .75 (observe) to .91 (describe; [47]). Test-retest reliability of the FFMQ facets is good to excellent ranging from .61 (act with awareness) to .84 (non-judgement; [47]).

\section{Resilience scale (RS-14)}

The Resilience Scale is a 14-item questionnaire which assesses five core features of resilience i.e. meaningful life, self-reliance, equanimity, perseverance, and coming home to yourself (existential aloneness). Resilience ratings for each feature are indicated through responses on a 7-point Likert scale ranging from 1 "strongly agree" to 7 "strongly disagree". The internal consistency of the scale has been described to be brilliant $(\alpha=.93)$ and it relates intensely $(\alpha=.97)$ with the complete version RS-25 [39].

\section{Personal well-being index -adult (PWI-A)}

[48] PWI-A is a 7-item questionnaire that assesses satisfaction with a range of different life areas that comprised standard of living, health, achievement in life, personal relationships, safety and spirituality. Satisfaction ratings for each domain are indicated through responses to a Likert scale from " 0 " not satisfied to " 10 " completely satisfied. The index has demonstrated excellent test-retest reliability (i.e., a coefficient of .84; [49].

\section{Procedure}

Research was approved by the Cairnmillar Human Research Ethics Committee. The study was conducted via online survey software (i.e. Survey Monkey). Participants were first asked to complete the demographic questionnaire (age and gender), and then the program introduced each of the psychological measures.

\section{Results}

\section{Preliminary analysis}

Prior to analysis, data screening and cleaning was performed using SPSS. This included the examination of variables (dependent \& inde- 
pendent) using frequency tables, reliability and internal consistency analysis; examination of the accuracy of data entry and missing values. Except for random omissions in survey response, the missing cases were deleted. Random omissions were determined using Little's MCAR test. For those determined to be randomly missing data, the values were replaced through expectation management.

Variables were then evaluated for normality, linearity, and univariate and multivariate outliers. This was done through analysis of descriptive statistics, graphical approaches and Shapiro-Wilk test. Values outside the range of -3.29 and +3.29 were considered as outliers. While Table 2: Means, Standard Deviations, Skewness and Kurtosis. there were several outliers identified amongst responses to the resilience questionnaire all such values were reasonable and were considered appropriate for analysis.

Tests for normality (Shapiro- Wilk test) showed significant $\mathrm{p}$ values for the resilience and mindfulness variables. This is not uncommon with bigger sample sizes [50]. However, histograms, scatter plots and Q-Q plot specified that the assumptions of normality, linearity and homoscedasticity were fulfilled. Descriptive information such as mean, standard deviations, skewness and kurtosis for the data is shown in Table 2.

\begin{tabular}{|c|c|c|c|c|}
\hline $\mathbf{N}=\mathbf{1 0 4}$ & $\mathbf{M}$ & SD & Skew & Kurt \\
\hline Resilience & 74.07 & 14.32 & -1.45 & 3.30 \\
\hline Mindfulness & 77.81 & 10.87 & .501 & .906 \\
\hline Well-being & 48.24 & 11.76 & -.335 & -.176 \\
\hline
\end{tabular}

\section{Principal component analysis}

Participants' answers on the FADNESS were scored based on their reported intensity. A score was assigned for every component of an item, apart from the null option (i.e., "I haven't been feeling this way"). When the null option was selected, the intensity for the other response alternatives (functional, dysfunctional, and functional+ dysfunctional) was entered as 0 . When any of the non-null options were selected, the reported intensity for the selected response was retained whereas the intensity for the other options were entered as 0 . Total scores were calculated for functional and dysfunctional subscales, by adding the values stated for each functional or dysfunctional emotion.
A principal component analysis (PCA) was conducted on the FADNES to confirm its factor structure. PCA was used on the all the items (10 items and 30 variables) of FADNES with a limitation of 2 factors. Varimax orthogonal rotation method was chosen to maximise the variance among the absolute values of loadings of each factor [50]. The Kaiser- Meyer-Olkin measure of sampling adequacy for the analysis was KMO $=.541$, which was acceptable. It was indicated by Barlett's test of sphericity, $\chi^{2}(435)=1041.275, \mathrm{p}<.001$, that correlations among items were adequate for PCA. The factor loadings for individual items are shown in Table 3.

Table 3:Factor loading for the FADNES items.

\begin{tabular}{|c|c|c|c|}
\hline \multirow[t]{2}{*}{ Item } & \multirow[t]{2}{*}{ Item's Type \& No. } & \multicolumn{2}{|c|}{ Component } \\
\hline & & 1 & 2 \\
\hline Upset + desperate & FD1 & .819 & -.092 \\
\hline Fearful +terrified & FD2 & .735 & -.024 \\
\hline Annoyed+furious & FD3 & .624 & -.102 \\
\hline Frightened +dreadful & FD4 & .615 & .409 \\
\hline Concerned +anxious & FD5 & .603 & .030 \\
\hline Scared +panicked & FD6 & .602 & .355 \\
\hline Sad +depressed & FD7 & .595 & -.200 \\
\hline Regret + guilt & FD8 & .499 & -.152 \\
\hline Dissatisfied +angry & FD9 & .438 & -.282 \\
\hline Sad & F1 & -.255 & .115 \\
\hline Angry & D1 & -.233 & .086 \\
\hline Guilt & D2 & -.214 & .150 \\
\hline Upset & F2 & -.177 & .039 \\
\hline Anxious & D3 & -.170 & -.021 \\
\hline Fearful & F3 & .138 & .703 \\
\hline Dissatisfied & $\mathrm{F} 4$ & .082 & .512 \\
\hline Concerned & F5 & -.137 & .450 \\
\hline Daunted & D4 & .410 & .450 \\
\hline Frightened & F6 & -.191 & .439 \\
\hline
\end{tabular}




\section{Psychology and Behavioral Science International Journal}

\begin{tabular}{|c|c|c|c|}
\hline Desperate & D5 & -.127 & .416 \\
\hline Scared & F7 & .022 & .379 \\
\hline Annoyed & F8 & -.307 & .365 \\
\hline Regret & F9 & .199 & .348 \\
\hline Dreadful & D6 & .162 & -.243 \\
\hline Helpless +daunted & FD10 & .145 & -.211 \\
\hline Helpless & F10 & -058 & .196 \\
\hline Panicked & D7 & -050 & .184 \\
\hline Furious & D8 & -.108 & .178 \\
\hline Terrified & D9 & .085 & .168 \\
\hline Depressed & D10 & .121 \\
\hline
\end{tabular}

Note: $\mathrm{F}=$ functional, $\mathrm{D}=$ dysfunctional, $\mathrm{FD}=$ functional $\&$ dysfunctional.

The two extracted factors were responsible for $23 \%$ of the variance. As displayed, the combined functional and dysfunctional item components came out as the maximum consistent factor (7 out of the 10 item components were loading on the first factor and the loadings were higher than .30). Two of the functional and dysfunctional items loaded on both factors whereas only one item had a low loading. A few functional item components loaded on the second factor and their highest loading was .70. Dysfunctional item-components had mostly low loadings and were spread across both factors.
It was decided to re-run the analysis after excluding the items that did not load above (.30) in line with suggested practice [50]. Items with cross loadings were also considered complex for further analysis and were excluded on that basis. Results demonstrated improved sampling adequacy, KM0 $=.629$. Similarly, Barletts' test of sphericity, $\chi^{2}(91)=$ $270.836, \mathrm{p}<.001$, identified that correlations among items were adequately large for PCA. The factor loadings for the individual items are shown in Table 4.

Table 4:Factor loading for the FADNES items.

\begin{tabular}{|c|c|c|c|}
\hline \multirow{2}{*}{ Item } & Item's type \& no. & $\mathbf{1}$ & \multicolumn{2}{|c|}{ Component } \\
\cline { 2 - 4 } & & .793 & .086 \\
\hline Upset +desperate & FD1 & .742 & -.073 \\
\hline Fearful + terrified & FD2 & -.016 \\
\hline Annoyed + Furious & FD3 & .592 & .024 \\
\hline Concerned+anxious & FD5 & .586 & .190 \\
\hline Sad+depressed & FD7 & .577 & -.213 \\
\hline Regret+guilt & FD8 & -.090 \\
\hline Dissatisfied+angry & FD9 & .535 & .679 \\
\hline Fearful & F3 & .460 & .532 \\
\hline Dissatisfied & F4 & .049 & .346 \\
\hline Concerned & F5 & -.303 & .523 \\
\hline Frightened & F6 & -.079 & .407 \\
\hline Desperate & D5 &. .229 & .550 \\
\hline Scared & F7 & -.010 & .406 \\
\hline Regret & F9 & .106 & \\
\hline
\end{tabular}

Note: $\mathrm{F}=$ functional, $\mathrm{D}=$ dysfunctional, $\mathrm{FD}=$ functional $\&$ dysfunctional.

The two factors extracted were, responsible for $34 \%$ of the variance. The first factor was comprised of the functional and dysfunctional items and was renamed, 'dysfunctional emotions'. This was in line with previous research that has shown that dysfunctional emotions often co-occur with their functional counterpart $[10,11]$. The second factor named as 'functional emotions' grouped together functional item com- ponents. The items contributing to the functional emotion list included one item, "Desperate", that had theoretically been a dysfunctional emotion. Total scores for functional and dysfunctional emotions were calculated by adding the scores of the items extracted through principal component analysis. Mean and standard deviation for each factor extracted are shown in Table 5.

Table 5:Mean and Standard Deviation functional and functional/dysfunctional items.

\begin{tabular}{|c|c|c|}
\hline & M & SD \\
\hline Upset +Desperate & .60 & 1.27 \\
\hline
\end{tabular}




\section{Psychology and Behavioral Science International Journal}

\begin{tabular}{|c|c|c|}
\hline Fearful + terrified & .29 & .92 \\
\hline Annoyed+ Furious & .29 & .89 \\
\hline Concerned+ anxious & .97 & 1.46 \\
\hline Sad + depressed & .69 & 1.21 \\
\hline Regret+ Guilt & .29 & .90 \\
\hline Dissatisfied+ angry & .57 & 1.15 \\
\hline Fearful & .34 & .83 \\
\hline Dissatisfied & .51 & 1.02 \\
\hline Concerned & .47 & 1.04 \\
\hline Frightened & .13 & .44 \\
\hline Desperate & .26 & .75 \\
\hline Scared & .27 & .71 \\
\hline Regret & .40 & .95 \\
\hline
\end{tabular}

\section{Mediation Analysis}

Structural equation modelling (SEM) framework was used to conduct the mediation analysis. SEM was preferred instead of commonly used mediation analysis procedures developed by Baron \& Kenny [51] because SEM allows for more complicated mediation models in a single analysis [52] and there was more than one independent variable and mediator used.
Mediating influences of mindfulness and resilience for the relationship between dysfunctional negative emotions and well-being were evaluated. Mediation analysis was performed in three steps. The first step assessed the relationship between the different emotional categories and well-being. This analysis shows that negative emotions explain $26 \%$ of the variance in well-being, with functional and dysfunctional negative emotions each contributing uniquely to reduction in well-being. The model of these relationships is shown in Figure 1.

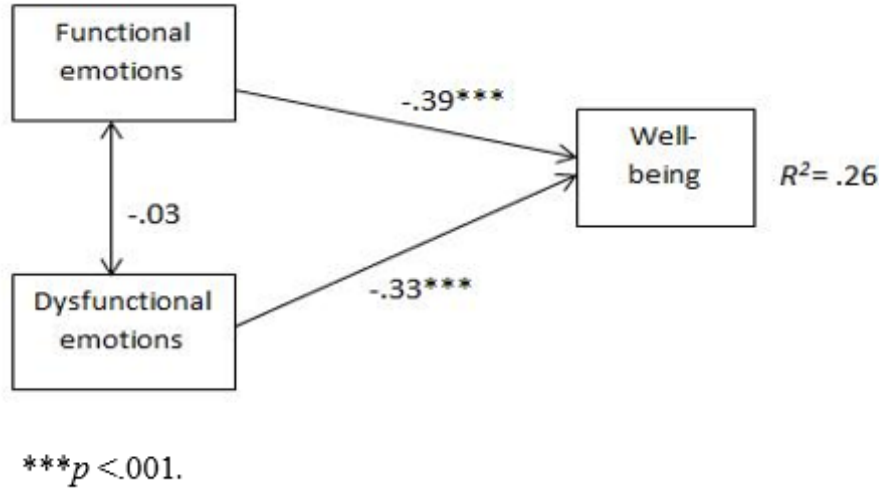

Figure 1: The total effect of functional and dysfunctional emotions on Well-being.

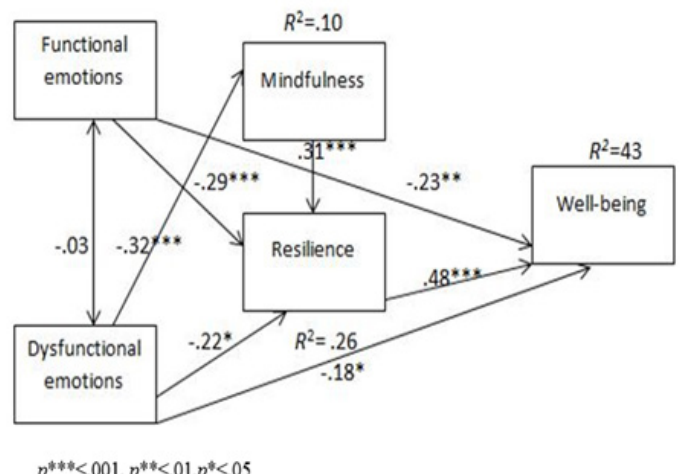

Figure 2: Partial mediation model of functional and dysfunctional emotions with well-being 
In the second step of the mediation analysis the direct effects of functional and dysfunctional negative emotions and the indirect effect through the mediators, were analysed. The results revealed partial mediation. The combination of direct and indirect effects accounted for $43 \%$ of variance in the DV. The model fit statistics indicated a reasonable model fit, Chi-square $=6.42, \mathrm{p}=.04$, Chi-square $/ \mathrm{df}=3.21, \mathrm{CFI}=.96$. $\mathrm{TLI}=.78, \mathrm{NFI}=.94, \mathrm{RMSEA}=.15, \mathrm{SRMR}=.06$. The direct and indirect effects of functional and dysfunctional emotions on well-being are presented in Figure 2.

The indirect effects of the emotional categories on well-being through the mediators was then analysed, after controlling for the direct effects. Results show that the relationship between the mediators and well-being improved. Indirect effects accounted for $37 \%$ of variance in the well-being. The results are shown in Figure 3.

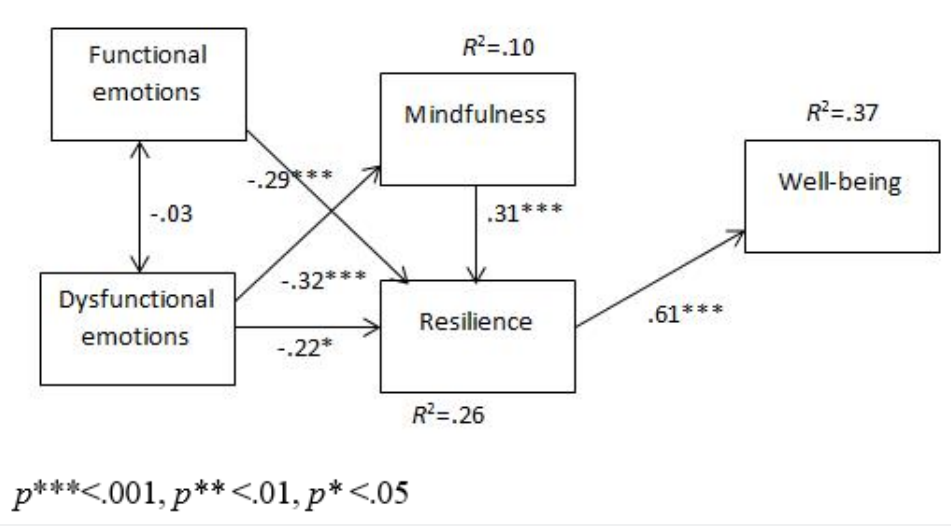

Figure 3: Indirect effects of functional and dysfunctional emotions on well-being.

\section{Discussion}

The present study investigated Ellis' [2] binary model of emotions. It examined the relationship between functional negative emotions, dysfunctional negative emotions, resilience, mindfulness and well-being. The PCA did demonstrate that there were two distinct groups of negative emotions reported by participants on the FADNES. The grouping was in line with the theoretical separation of emotions into a group of functional emotions and a group of combined functional and dysfunctional emotions. No distinct factor with only dysfunctional negative emotions was found.

The hypothesis that functional negative emotions and dysfunctional negative emotions would be associated with reduced well-being was then investigated. This was supported. Both forms of negative emotions uniquely contributed to a reduction in well-being. Path analysis then demonstrated support for the proposed mediators on these relationships. That is, mindfulness and resilience were found to in series mediate the relationship between dysfunctional negative emotions and well-being, while resilience alone mediated the relationship between functional negative emotions and well-being.

\section{Distinguishing between functional and dysfunctional emo- tions}

The analysis of the FANDES, through the PCA, finding a distinct group of functional emotions and a group of functional-dysfunctional emotions was like that reported by Mogoase \& Stefan [11]. This demonstrated that there was a factor made up primarily of the items considered to be representing functional emotions and a second made up of the combined functional-dysfunctional emotions. The emotional terms considered to represent dysfunctional negative emotions were mostly spread out, loading on both functional and the functional-dysfunctional factors. Unlike Mogoase and Stefan, a second PCA was conducted on statistical grounds, rather than theoretical grounds. This resulted in the inclusion of one of the proposed items for dysfunctional emotions (i.e. desperate) being included into the functional emotion factor. It is possible that the meaning of the term desperate has become a common term to express daily worries, however further research is required to clarify this issue.

These results suggest that people do not methodically recognise a difference between functional and dysfunctional negative emotions when single emotion labels are presented to them. They also suggest that while functional negative emotions may occur in isolation, dysfunctional emotions may not. That is when a dysfunctional emotion is activated; the functional version of the emotion also appears to be activated. This is consistent with other studies into the binary model of emotions by David et al. [10] and Mogoase \& Stefan [13].

A further implication of the current findings is that functional and dysfunctional negative emotions establish two qualitatively dissimilar mechanisms of negative affect (i.e., they loaded on distinct factors). This supports the binary model of distress proposed by Ellis [2]. It is also consistent with past investigations of the binary model $[10,11]$.

\subsection{Dysfunctional emotions and well-being}

The finding that dysfunctional negative emotions predicted well-being, with an increase in dysfunctional emotions being associated with reduced well-being provides further support of Ellis's model of distress. He theorised that dysfunctional negative emotions are associated with negative subjective experience, irrational beliefs and dysfunctional behavioural responses [2]. This study found that those 
experiencing dysfunctional negative emotions experienced the greatest reduction to their well-being, remembering that such individuals reported experiencing a combination of dysfunctional and functional negative emotions, thereby receiving the detrimental influence of both forms of negative emotions to their well-being.

The specific relationship between dysfunctional negative emotions and well-being was then found to be mediated through a pathway of resilience and mindfulness. That is, those reporting more dysfunctional emotions reported lower levels of mindfulness, which in turn led to lower levels of resilience and lower levels of well-being. After introducing the mediators, the direct effect of the dysfunctional negative emotions was reduced, however it was still present. Results also showed that once the direct effects of dysfunctional negative emotions were controlled, the relationship between resilience, mindfulness and well-being was further improved.

These findings could have important treatment implications. It indicates that it can be advantageous to improve an individual's resilience and mindfulness skills as an approach to managing the impact of dysfunctional negative emotions upon well-being. This is in line with research that has demonstrated that higher levels of mindfulness are associated with improved emotion regulation, decreased stress, and improved coping [12,23,24,53].

The current research also demonstrates a pathway through which mindfulness-based interventions can mitigate the impact of dysfunctional negative emotions upon well-being. It indicates that mindfulness protects against the impact of dysfunctional emotions. This then promotes increased resilience, which has been shown to include the ability to effectively regulate emotions [37] and the skill to take positive meaning out of a difficult situation $[40,54]$. These findings support the idea that mindfulness promotes people to disengage from instinctive processes and to accept as well as tolerate their emotions, with subsequent benefits to well-being [20]. Together it suggests that people with higher levels of mindfulness are more resilient in the face of challenges that trigger dysfunctional emotional experiences.

\section{Functional emotions and well-being}

As hypothesized, functional negative emotions predicted the level of well-being, with an increase in functional negative emotions resulting in reduced well-being. This result is not unexpected as according to binary model of distress, rational beliefs enable a person to evaluate a situation as moderately negative, and that the person will then experience functional negative emotions. These emotions are then suggested to help the individual to deal with the situation through an open-minded approach, accepting the problem and staying engaged with it to work on personal goals [55]. This implies that functional negative emotions are not an absence of negative emotions and could still thereby have a negative impact on mood levels and well-being to a certain extent. Therefore, a relationship between functional negative emotions and reduced well-being is justified.

The mediation analysis indicated that functional negative emotions negatively affected resilience, which in turn led to a reduction in well-being. Unlike dysfunctional negative emotions, this was not medi- ated by mindfulness. The absence of a relationship between functional negative emotions and mindfulness is consistent with the research by Mogoase \& Stefan [11], who found no relationship between functional negative emotions and rational thoughts.

The negative relationship between resilience and functional negative emotions is somewhat inconsistent with the argument that functional negative emotions do not negatively impact functionality [8]. One possible explanation could be that as the study was cross-sectional, while people experienced a reduction in resilience with functional negative emotions, this is not related to their capacity to act mindfully, so in the longer term they may be able to better recover and maintain their resilience and well-being. Maintaining the capacity to be mindful may also help the individual avoid the use of dysfunctional coping strategies (e.g. suppression, avoidant coping), which have been found to contribute to further mental health difficulties $[56,57]$.

\section{Limitations}

The ability to generalise the findings of this study is limited by several factors. Firstly, the participants were from a limited range of ethnic backgrounds. A more diverse sample would have promoted the generalizability of the outcomes to bigger populations. The measures used within the study may also have influenced the outcomes. While each scale was selected based on its validity and reliability there is still controversy over some of these (e.g. the FFMQ; [47]). Furthermore, the FADNES is a newly developed scale and its utility in assessing for functional and dysfunctional emotions is still questionable. It covers a limited number of possible emotions and relies on self-report. It also relies on a nuanced understanding of emotional terminology. This may result in difficulties for those with a limited emotional vocabulary differentiating their experiences, and this is a factor that has been linked to emotion regulation $[58,59]$. Hence the scale might not be representative of other functional and dysfunctional negative emotions and some individuals may not differentiate between the terms used. It is also important to note that the two factors in the revised principle component analysis only accounted for $34 \%$ of the variance, which is lower than the suggested threshold of $60 \%$ for a valid construct [60].

Online questionnaires were used for data collection and therefore the information was completely dependent on self-report instruments and control over the participants in this procedure is less than the experimental conditions. Hence it is hard to predict if the participants were able to relate to their past week's emotional experiences. Furthermore, there is a possibility that a tendency towards suppressive ways of coping with negative emotions could have led some participants to pick responses that minimized their psychological distress [61].

\section{Implications for Future Research}

In future studies, a variety of approaches could be used to deal with these limitations. For instance, causality could not be determined from these results because the present study was a cross-sectional design. Hence, for a more precise assessment of mediation effect of mindfulness and resilience, longitudinal and experimental designs are desirable in further work. The participants were not representative of a clinical sample thus, current results could not be generalised to clini- 
cal populations. Future research should focus on replicating this study on a clinical sample as this could provide further valuable information on the qualitative distinction between negative emotions. A qualitative component such as interviewing the participants and getting their firsthand perspectives on the constructs of the present study is an opportunity for future research.

Upcoming studies should replicate these findings using the version of the FADNES that includes only functional and functional + dysfunctional items. A revised version of the FADNES scale should also include additional negative emotions and linguistic descriptors of emotions (e.g., resentment, jealousy, envy, greed, insensitivity etc.). Inclusion of a survey about recalling a stressful event in the past week or participants' beliefs (rational/ irrational) and associated cognitions (helpful/ unhelpful) regarding stressful situations before filling in the FADNES, could also be supportive for them to make a better distinction on quality of their experienced emotions. This would lead to an improved selection of the content and, subsequently better psychometric properties. The percentage of variance explained through the extracted components of PCA was somewhat moderate. Future studies could also investigate the reasons behind the modest variance explained by the functional and dysfunctional negative emotions and examine the factors that could explain the rest of the variance. It would be beneficial to further study the differences in how functional and dysfunctional emotions relate to other mental health constructs not covered in this study.

\section{Conclusion}

In conclusion, the findings of the current study provided further support for the binary model of distress [2]. This suggests that within the realm of negative emotions there exist both functional and dysfunctional emotions. Results also suggest that these different types of negative emotions are not mutually exclusive and co-occur [62-66]. The results suggest that functional negative emotions are not as harmful as dysfunctional negative emotions to other psychological processes. This has considerable implications for the theoretical conceptualization of distress and the clinical goals for mental health interventions. It further suggests that treatment interventions focused on improving mindfulness and resilience skills are well suited to reduce the impact of dysfunctional negative emotions upon well-being. Future research to replicate these findings and to develop a more detailed understanding of the binary model would also appear to be warranted [67-69].

\section{References}

1. Cohn MA, Fredrickson BL, Brown SL, Mikels JA, Conway AM (2009) Happiness unpacked: positive emotions increase life satisfaction by building resilience. Emotion 9(3): 361-368.

2. Ellis A (1994) Reason and emotion in psychotherapy (rev. ed.) Birch Lane, Secaucus, NJ, US State.

3. Hayes S (2004) Acceptance and Commitment Therapy and the new behavior therapies: Mindfulness, acceptance and relationship. In: $\mathrm{S}$ Hayes, $\mathrm{V}$ Follette, $\mathrm{M}$ Linehan (Eds.), Mindfulness and acceptance: Expanding the cognitive-behavioral tradition. Guildford Press, New York, USA, pp. 1-30.

4. Ryff CD, Singer B (2003) Flourishing under fire: Resilience as a prototype of challenged thriving. In: CLM Keyes, J Haidt (Eds.), Positive psychology and the life well-lived. APA. Washington DC, USA, p. 15-36.
5. Teasdale JD (1999) Metacognition, mindfulness and the modification of mood disorders. Clinical Psychology \& Psychotherapy 6(2): 146155.

6. Hyland P, Boduszek D (2012) Resolving a difference between cognitive therapy and rational emotive behaviour therapy: towards the development of an integrated CBT model of psychopathology. Mental Health Review Journal 17(2): 104-116.

7. Russell JA, Carroll JM (1999) On the bipolarity of positive and negative affect. Psychol Bull 125(1): 3-30.

8. Ellis A, DiGiuseppe R (1993) Are inappropriate or dysfunctional feelings in rational-emotive therapy qualitative or quantitative? Cognitive Therapy and Research 5: 471- 477.

9. Kassinove H, Eckhardt C, Endes R (1993) Assessing the intensity of "appropriate" and "inappropriate" emotions in rational-emotional therapy. Journal of Cognitive Psychotherapy: International Quarterly 7: 227-239.

10. David D, Montgomery GH, Macavei B, Bovbjerg D (2005) An empirical investigation of Albert Ellis' binary model of distress. J Clin Psychol 61(4): 499-516.

11. Mogoase C, Stefan S (2013) Is there a difference between functional and dysfunctional negative emotions? The preliminary validation of the functional and dysfunctional negative emotions scale. Journal of Cognitive and Behavioral Psychotherapies 13(1): 13-32.

12. Brown KW, Ryan RM, Creswell JD (2007) Addressing fundamental questions about mindfulness. Psychological inquiry 18: 272-281.

13. Hulsheger UR, Hugo JEM, Alberts Feinholdt A, Lang JWB (2013) Benefits of mindfulness at work: The role of mindfulness in emotion regulation, emotional exhaustion, and job satisfaction. J Appl Psychol 98(2): 310-325.

14. Kostanski M, Hassed C (2008) Mindfulness as a concept and a process. Australian Psychologist 43: 15-21.

15. Siegel DJ (2007) Mindfulness training and neural integration: differentiation of distinct streams of awareness and the cultivation of well-being. Journal of Social, Cognitive and Affective Neuroscience 2: 259-263.

16. Germer CK, Siegel RD, Fulton PR (2005) Mindfulness and psychotherapy. Guildford Press, New York, USA.

17.Germer C (2009) The mindful path to self-compassion: Freeing yourself from destructive thoughts and emotions. Guildford Press, New York, USA.

18. Baer RA (2006) Using self-report assessment methods to explore facets of mindfulness. Assessment 13: 27-45.

19. Hayes SC (2002) Acceptance, mindfulness, and science. Clinical Psychology: Science and Practice 9(1): 101-106.

20. Alberts HJEM, Schneider F, Martijn C (2012) Dealing efficiently with emotions: Acceptance-based coping with negative emotions requires fewer resources than suppression. Cogn Emot 26: 863-870.

21. Marcks BA, Woods DW (2005) A comparison of thought suppression to an acceptance-based technique in the management of personal intrusive thoughts: A controlled evaluation. Behav Res Ther 43(4): 433-445.

22. Langer EJ, Moldoveanu M (2000) The construct of mindfulness. Journal of Social Issues 56: 1-9.

23. Mennin DS, McLaughlin KA, Flanagan TJ (2009) Emotion regulation deficits in generalized anxiety disorder, social anxiety disorder, and their co-occurrence. J Anxiety Disord 23: 866-871.

24. Tugade MM, Fredrickson BL (2004) Resilient individuals use positive emotions to bounce back from negative emotional experiences. J Pers Soc Psychol 86(2): 320. 
25. Bishop SR, Lau M, Shapiro S, Carlson L, Anderson ND, et al. (2004) Mindfulness: A proposed operational definition. Clinical Psychology: Science and Practice 11(3): 230-241.

26. Frye L, Spates CR (2012) Prolonged Exposure, Mindfulness, and Emotion Regulation for the Treatment of PTSD. Clinical Case Studies 11(3): 184-200.

27. Eifert GH, Forsyth JP (2005) Acceptance and commitment therapy for anxiety disorders: A practitioner's treatment guide to using mindfulness, acceptance, and values-based behavior change strategies. Guilford Press, New York, USA.

28. Cahn BR, Polich J (2009) Meditation (Vipassana) and the P3a eventrelated brain potential. Int J Psychophysiol 72: 51-60.

29. Goldin PR, Gross JJ (2010) Effects of mindfulness-based stress reduction (MBSR) on emotion regulation in social anxiety disorder Emotion 10: 83-91.

30. Moore A, Malinowski P (2009) Meditation, mindfulness and cognitive flexibility. Conscious Cogn 18: 176-186.

31. Ortner CNM, Kilner SJ, Zelazo PD (2007) Mindfulness meditation and reduced emotional interference on a cognitive task. Motivation and Emotion 31: 271-283.

32. Bruce A, Young L, Turner L, Vander Wal R, Linden W (2002) Meditationbased stress reduction: Holistic practice in nursing education. In: LE Young, VE Hayes (Eds.), Transforming health promotion practice: Concepts, issues, and applications, Davis, Victoria, British Columbia, Canadian Province, pp. 241-252.

33. Carmody J (2009) Evolving conceptions of mindfulness in clinical settings. Journal of Cognitive Psychotherapy: An International Quarterly 23: 270-280.

34. McKim RD (2008) Rumination as a mediator of the effects of mindfulness: Mindfulness-based stress reduction (MNSR) with a heterogeneous community sample experiencing anxiety, depression, and/or chronic pain. Dissertation Abstracts International: Section B: The Sciences and Engineering 68: 7673.

35. Rosenzweig S, Reibel DK, Greeson JM, Brainard GC, Hojat M (2003) Mindfulness-based stress reduction lowers psychological distress in medical students. Teach Learn Med 15: 88-92.

36. Shapiro SL, Schwartz GE, Bonner G (1998) Effects of mindfulnessbased stress reduction on medical and premedical students. Journal of Behavioral Medicine 21: 581-599.

37. Ahern NR, Kiehl EM, Sole ML, Byers J (2006) A review of instruments measuring resilience. Issues Compr Pediatr Nurs 29: 103-125.

38. Lazarus R (1993) From psychological stress to the emotions: A history of changing outlooks. Annu Rev Psychol 44: 1-21.

39. Wagnild G (2010) Discovering your resilience core. Most.

40. Affleck G, Tennen H (1996) Construing benefits from adversity: Adaptational significance and dispositional underpinnings. J Pers 64 899-922.

41. Ong AD, Bergeman CS, Boker SM (2009) Resilience comes of age: defining features in later adulthood. J Pers 77(6): 1777-1804.

42. Billings DW, Folkman S, Acree M, Moskowitz JT (2000) Coping and physical health during caregiving: the roles of positive and negative affect. J Pers Soc Psychol 79(1): 131-142.

43. O'Rourke N (2004) Psychological resilience and the well-being of widowed women. Ageing International 29(3): 267-280.

44. Keye MD, Pidgeon AM (2013) Investigation of the Relationship between Resilience, Mindfulness, and Academic Self-Efficacy. Open Journal of Social Sciences 1(6): 1-4
45. Orzech KM, Shapiro SL, Brown KW, McKay M (2009) Intensive mindfulness training-related changes in cognitive and emotional experience. The Journal of Positive Psychology 4: 212-222.

46. Kuyken W, Byford S, Taylor RS, Watkins E, Holden E, et al. (2008) Mindfulness-based cognitive therapy to prevent relapse in recurrent depression. J Consult Clin Psychol 76: 966-978.

47. Bohlmeijer E, Klooster PM, Fledderus M, Veehof M, Baer R (2011) Psychometric properties of the five facet mindfulness questionnaire in depressed adults and development of a short form. Assessment;18(3): 308-320.

48. International (4) Personal Wellbeing Index ( $4^{\text {th }}$ Edn), Melbourne: Australian Centre on Quality of Life, Deakin University, Australia.

49. Lau ALD, Cummins RA (2005) Test-retest Reliability of the Personal Wellbeing Index. Unpublished Research Report, The Hong Kong Polytechnic University, Hong Kong, China.

50. Field A (2009) Discovering statistics using SPSS. SAGE Publications Ltd, London, UK.

51. Baron RM, Kenny DA (1986) The Moderator-Mediator Variable Distinction in Social Psychological Research - Conceptual, Strategic, and Statistical Considerations. J Pers Soc Psychol 51(6): 1173-1182.

52. Gunzler D, Chen T, Wu P, Zhang H (2013) Introduction to mediation analysis with structural equation modeling. Shangai Arch Psychiatry 25(6): 390-394.

53. Shapiro SL, Brown KW, Biegel GM (2007) Teaching self-care to caregivers: Effects of mindfulness-based stress reduction on the mental health of therapists in training. Training and Education in Professional Psychology 1: 105-115.

54. Anderson KJ (1994) Impulsivity, caffeine, and task difficulty: A withinsubjects test of the Yerkes- Dodson law. Personality and Individual Differences 6: 813-829.

55. Opris D, Macavei B (2005) The distinction between functional and dysfunctional negative emotions: An empirical analysis. Journal of Cognitive and Bahavioral Psychotherapies 5: 181-195

56. Dobson DJG, Dobson KS (2018) Avoidance in the clinic: Strategies to conceptualize and reduce avoidant thoughts, emotions, and behaviors with cognitive-behavioral therapy. Practice Innovations 3(1): 32-42.

57.Pittig A, Treanor M, LeBeau RT, Craske MG (2018) The role of associative fear and avoidance learning in anxiety disorders: Gaps and directions for future research. Neurosci Biobehav Rev 88: 117-140.

58. Barrett LF, Gross J, Christensen TC, Benvenuto M (2001) Knowing what you're feeling and knowing what to do about it: Mapping the relation between emotion differentiation and emotion regulation. Cognition and Emotion 15(6): 713-724.

59. Kashdan TB, Barrett LF, McKnight PE (2015) Unpacking emotion differentiation: Transforming unpleasant experience by perceiving distinctions in negativity. Current Directions in Psychological Science 24(1): 10-16.

60. Hair JF, Black WC, Babin BJ, Anderson RE (2018) Multivariate Data Analysis. Cengage Learning.

61. Carver CS, Scheier MF, Weintraub JK (1989) Assessing coping strategies: A theoretical based approach. J Pers Soc Psychol 56(2): 267-283.

62. International Wellbeing Group (2006) Personal Wellbeing Index ( $4^{\text {th }}$ Edn), Australian Centre on Quality of Life, Deakin University. Melbourne, Australia.

63. Lawford J, Eiser C (2001) Exploring links between the concepts of quality of life and resilience. Pediatr Rehabil 24(4): 209-216. 
64. Barrett LF, Gross J, Christensen TC, Benvenuto M (2001) Knowing what you're feeling and knowing what to do about it: Mapping the relation between emotion differentiation and emotion regulation. Cognition and Emotion 15(6): 713-724.

65. Dryden W, Digiuseppe R, Neenan M (2003) A Primer on Rational Emotive Behavior Therapy.

66. Hair JF, Black WC, Babin BJ, Anderson RE (2018) Multivariate Data Analysis. Cengage Learning.
67. Lau ALD, Cummins RA (2005) Test-retest Reliability of the Personal Wellbeing Index. Unpublished Research Report, The Hong Kong Polytechnic University, Hong Kong, China.

68. Wagnild G (2009) A Review of the Resilience Scale. J Nurs Meas 17(2):105-113.

69. Yerkes RM, Dodson JD (1908) The relation of strength of stimulus to rapidity of habit formation. Journal of Comparative Neurology and Psychology 18: 459-482.

\section{Your next submission with Juniper Publishers will reach you the below assets}

- Quality Editorial service

- Swift Peer Review

- Reprints availability

- E-prints Service

- Manuscript Podcast for convenient understanding

- Global attainment for your research

- Manuscript accessibility in different formats

( Pdf, E-pub, Full Text, Audio)

- Unceasing customer service

Track the below URL for one-step submission https://juniperpublishers.com/online-submission.php 